BOLETIN DE LA SOCIEDAD GEOLOGICA MEXICANA

V. LIII, (2000) 72-83

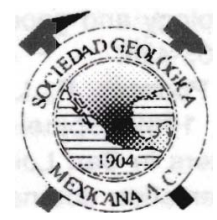

http://www.igeofcu.unam.mxIsgml

\title{
Volcanismo y extensión en la Provincia Extensional del Golfo de California
}

\author{
Arturo Martín Barajas \\ Departamento de Geología, CICESE, Km 107 carretera Tijuana-Ensenada, Ensenada, B. C. \\ amartin@cicese.mx
}

Resumen

La evolución del volcanismo en la Provincia Extensional del Golfo refleja el cambio de un régimen de subducción al desarrollo de un rift continental y a un rift oceánico con transferencia de la península a la Placa Pacífico durante el Mioceno tardfo-Plioceno. Una etapa de extensión temprana (25-12 Ma) en posición intraarco y trasarco en Sonora y en la margen oriental del Golfo ocurrió sincrónicamente con la última etapa del arco miocénico. En Sonora central la mayor extensión ocurrió en dos ó tres pulsos discretos entre -25 y -10 Ma, mientras que en la península de Baja California las primeras evidencias de extensión están definidas con edades radiométricas de $11 \mathrm{Ma}$ a $16 \mathrm{Ma}$. En Baja California Sur la mayor subsidencia de las cuencas marginales se reporta durante el Plioceno, aunque datos geocronológicos recientes sugieren una mayor edad (7-11 Ma) en la cuenca de Santa Rosalfa y por el levantamiento del bloque de Los Cabos.

El volcanismo posteriora la subducción muestra una diversificación importante en la composición y en el estilo eruptivo. En la penfnsula incluye flujos de lava alcalina y subalcalina de $<12$ Ma que cubren localmente a ignimbritas y andesitas subordinadas de 14 a $11 \mathrm{Ma}$. En Sonora, lavas basálticas intercaladas en depósitos continentales definen el inicio de la extensión en el Mioceno temprano, sin embargo, el volcanismo alcalino de tipo intraplaca se manifiestó hasta el Mioceno tardfo-Plioceno con volúmenes relativamente pequeños. A partir de -10 Ma basaltos y andesitas de afinidad toleitica fueron emplazados localmente en la costa de Sonora y en la penfnsula de Baja California sobre una región más amplia durante la etapa del protogolfo, este tipo de volcanismo subalcalino ocurre en los centros de dispersión y las zonas adyacentes a partir de la etapa moderna del Golfo. Desde la terminación de la subducción (ca. $12 \mathrm{Ma}$ ) el volcanismo calcialcalino ha ocurrido intermitentemente en las márgenes y algunas islas del Golfo, ocurre en estratovolcanes andesftico-dacftico (e.g. Tres Vfrgenes, Mencenares) y calderas (e.g. La Reforma, Puertecitos, Sierra Pinta), que imitan la actividad orogénica del Mioceno temprano. En la franja costera de Sonora el emplazamiento de domos y tobas riolfticas culminó hacia los $11 \mathrm{Ma}$.

La variación en la composición y en el estilo eruptivo del volcanismo post-subducción «12 Ma) refleja más una heterogeneidad de la fuente debida a un período prolongado de actividad orogénica previo a la distensión y a diferentes grados de fusión parcial relacionados con la inestabilidad térmica que ocasionó la distensión corticaly el desarrollo de un rift oceánico incipiente desde el Plioceno tardío.

Abstract

Volcanism in the Gulf of California Extensional Province (GEP) records the transition from subduction 
to continental rifting (15-4 Ma), and to oceanic rifting in the last 3.5 Ma. Early extension in central Sonora (25$12 \mathrm{Ma}$ ) is geometrically located in intra-arc and back-arc position relative to the Miocene volcanic are along eastern Baja California. Here beginning of extension is constrained between 11 and $16 \mathrm{Ma}$, as waning subduction and arc-volcanism in Baja California progressively ceased. In southem Baja Califomia the Santa Rosalía basin and uplift of the crystalline block La"Paz-Los Cabos region may have OCCU"ed in late Miocene and late-middle Miocene, respectively.

The transition from subduction to rifting is accompanied by a change in the eruptive processes and magma composition from dominantly calc-alkaline dacitic-andesitic volcaniclastic aprons to alkaline and tholeiitic basalt and andesite flows. Coeval calc-alkaline rhyolite to dacite dome complexes, caldera-type ignimbrite deposits, and composite andesitic volcanoes occur along the eastern margin of the Baja Peninsula and several islands within the Gulf. From -12 Ma up to present alkali basalt erupted intermittently in Baja Califomia to the west o, the main Gulf escarpment, and to a lesser extent, on the opposite side in central Sonora. In Plio-Quatemary times more typical intra-plate alkaline basalt have erupted in a few places on both rift sides. Low-K sub-alkaline basalt, and differentiates erupted from 10 to $5 \mathrm{Ma}$ along both margins and over a broad region in southem Baja California. Later «5 Ma), these sub-alkaline rocks erupted in a more restricted area within the Gulf, and evolved into MORB-like lavas in the nascent spreading centers.

The diversity in magma composition and eruptive style in post-subduction lavas may reflect heterogeneity in the mantle source due to long-lived subduction and various degree of partial melting during crustal extension.

\section{INTRODUCCiÓN}

La Provincia Extensional del Golfo de California comprende la región al oeste de la Sierra Madre Occidental hasta el escarpe principal del golfo en la península de Baja California (Figura 1). Este escarpe topográfico separa el macizo peninsular de una delgada franja en la porción oriental de la Península estructuralmente ligada a la Provincia Extensional del Golfo. Su evolución geológica es compleja debido a que registra durante el Neógeno el cambio en el régimen tectónico, de subducción a distensión cortical (rifting) y formación de piso oceánico en los últimos 3-4 Ma en algunas cuencas del Golfo. La causa de la distensión temprana en la provincia de Cuencas y Sierras (Basin and Range) es aún objeto de discusión, como también lo es la relación temporal causa-efecto del magmatismo cenozoico con el proceso distensivo; aunque existe consenso en que la extensión en la provincia de Cuencas y Sierras generó fusión parcial de manto litosférico y/o de la corteza inferior (c.f. Metcalf y Smith, 1995 y otros trabajos citados). En su etapa actual de apertura oceánica las cuencas de la mitad sur del Golfo han generado verdadera corteza oceánica.

El objetivo de este trabajo es presentar una síntesis basada en los trabajos publicados sobre el inicio y la evolución espacial y temporal de la distensión en la Provincia Extensional del Golfo de California y presentar las características geoquímicas principales del volcanismo durante el proceso de extensión. Principalmente se hace referencia a los trabajos que aportan datos recientes sobre las relaciones cronoestratigráficas de rocas volcánicas con el proceso distensivo, sus afinidades geoquímicas y la edad del levantamiento de bloques cristalinos durante el desarrollo de la Provincia Extensional del Golfo. La revisión bibliográfica sobre estos temas no es exhaustiva, sin embargo, se ha intentado utilizar como referencia las contribuciones más recientes y los trabajos que mayormente han impactado el conocimiento sobre el origen y las causas del magmatismo del Neógeno del proceso distensivo en la Provincia Extensional del Golfo.

\section{INICIO DE LA DISTENSIÓN}

La distensión cenozoica parece definir un patrón con edades más jóvenes de oriente a poniente a través de la Provincia Extensional del Golfo (Gans, 1997; Lee et al., 1996). En Sonora, el proceso de distensión cortical se manifestó después de un pulso de actividad volcánica hacia los $30 \mathrm{Ma}$ en la Sierra Madre Occidental (Ferrari et al., 1999). El volcanismo de arcO migró hacia el poniente, manteniendo una actividad importante durante el período de 23 a $12 \mathrm{Ma}$ a lo largo del margen oriental de la península de Baja Califomia (Figura 2) y en la costa de Sonora (Mora-Álvarez, 1993; MoraÁlvarez y McDowell, en prensa; Hausback, 1984; Sawlan y Smith, 1984; Martín-Barajas et al., en prensa). Durante este intervalo de tiempo, el proceso de máxima extensión en Sonora se desarrolló en una posición intraarco y trasarco con respecto a la actividad volcánica del arco miocénico en Baja California (Figura 2).

En Sonora central y en la porción noreste la formación de cuencas continentales y el desarrollo de núcleos metamórficos ocurrió principalmente entre 25 y 12 Ma (Nourse et al., 1994; McDowell et al., 1997; Gans, 1997). En la porción sur del estado, las 


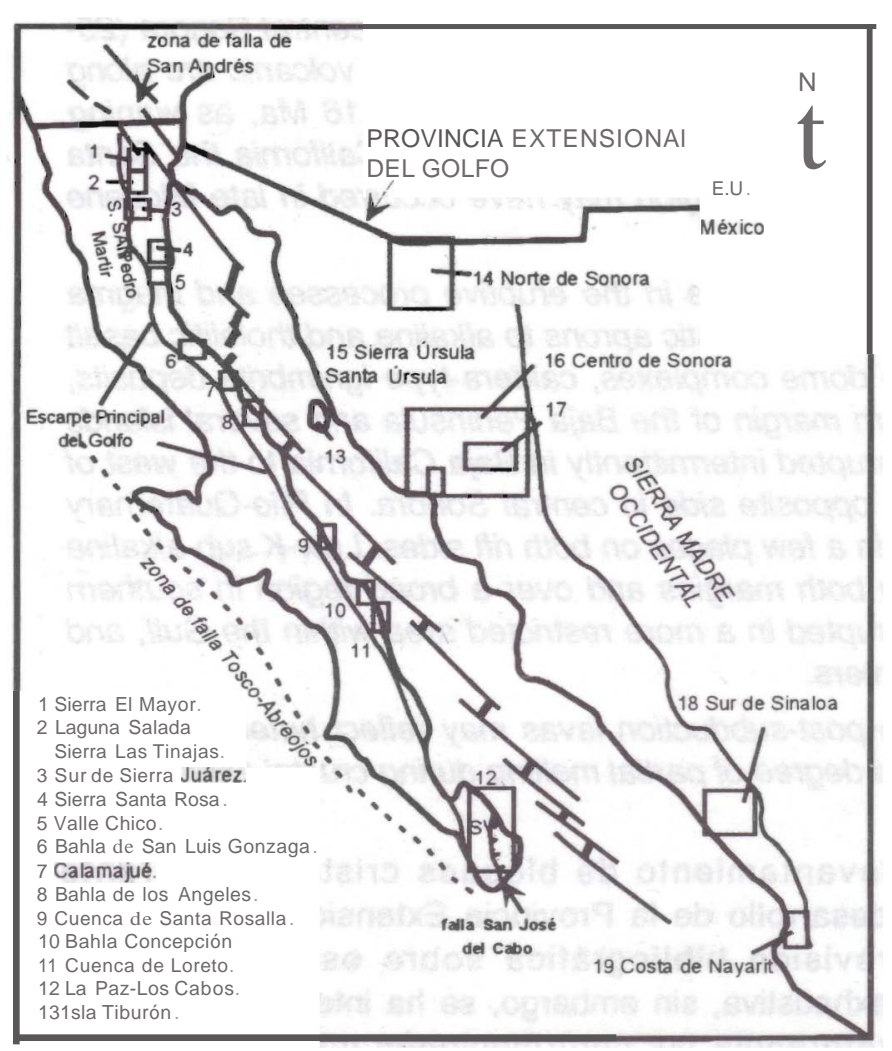

Figura 1. Provincia Extensional del Golfo y ubicación de las áreas con trabajos que han definido el inicio y la duración de la distensión en esta provincia. Las referencias a las localidades son: 1. Axen et al., en revisión en Tectonics; 2. Mendoza-Borunaa et al., 1998; 3. Lee et al., 1996; 4. Bryant, 1996; 5. Stock, 1989; 6. Martín-Barajas et al., en prensa; 7. Parkin, 1998; 8. Delgado Argote et al., en prensa; Delgado Argote, en revísión; 9. Holt et al., en prensa; Angelier et al., 1981; 10. McLean, 1988; 11. Umhoefer et al., 1994; 12. Fletcher et al., 1997; 1998; 13. Calmus et al., 1998; 14. Nourse et al., 1994; 15. Mora-Álvarez, 1993; 16. McDowell et al., 1997; 17. Gans, 1997; 18. Henry y Aranda-Gomez, en prensa; SV Sierra La Victoría. La zona de fractura Tosco-Abreojos es según Spenser y Normark. (1989)

relaciones cronoestratigráficas y estructurales en la secuencia volcánica terciaria sugieren que la mayor parte de la extensión entre la costa y el borde de la Sierra Madre Occidental también ocurrió en el intervalo de 27 a 12 Ma (Henry y Aranda-Gómez, 1992). Nuevos datos geocronológicos y estructurales en Sinaloa y Durango sugieren que la extensión del protogolfo (12-5 Ma) se manifestó sobre una zona más amplia a ambos lados de la Sierra Madre Occidental (Henry y ArandaGómez, en prensa) (Figura 3). Sin embargo, falta evaluar si otras regiones de Sonora central han experimentado extensión durante ese período de tiempo, ya que los estudios no incluyen la componente de extensión en el Plio-Cuaternario en la costa de Sonora y en la zona central.

Otras evidencias en Sonora sugieren una edad mayor (>28 Ma); como el fechamiento isotópico de milonitas en-fallas de bajo ángulo (detachment) en

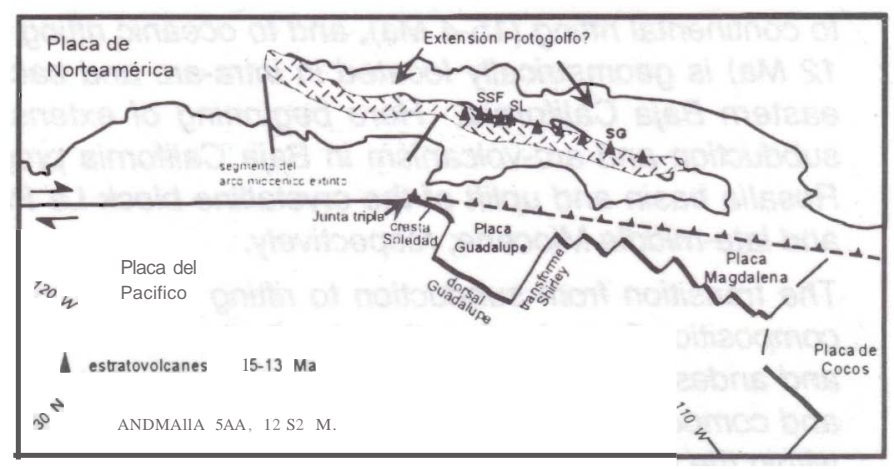

Figura 2. Marco tectónico del margen occidental de la península en el Mioceno Medio (modificado de Stock y Lee, 1994). La posición de la península corresponde a la reconstrucción previa a la apertura del Golfo.

Sonora central (Herrera-Urbina et al., 1995) y edades de enfriamiento con huellas de fisión en Isla Tiburón (Calmus et al., 1998). Estas evidencias indicarían que la extensión en Sonora pudo haber iniciado desde el Oligoceno, como se ha documentado al este de la Sierra Madre Occidental (c.f Henry y Aranda-Gómez, 1992) (Figura 3), sin embargo, el origen de este proceso de extensión temprana es aún tema de debate.

El inicio de la distensión en la margen oriental de la península es posterior al inicio de la distensión en Sonora y concuerda, además, con la terminación del volcanismo de arco y la subducción de la Placa Guadalupe hacia los $12 \mathrm{Ma}$ (Figura 2) (Stock y Lee, 1994; Lee et al., 1996). En varias localidades estudiadas en la mitad norte de la península las fallas en el escarpe del Golfo cortan a los depósitos más jóvenes del arco volcánico, los cuales son internamente concordantes (pretectónicos) y presentan edades mínimas de entre 15 y 16 Ma (Stock y Lee, 1994; Martín-Barajas et al., en prensa).

Las localidades con estudios cronoestratigráficos y estructurales indican que la edad del inicio de la distención en la península es posterior a 12 Ma (Figura 4). En el sur de Sierra Juárez las fallas del escarpe cortan a flujos basálticos fechados en $<11 \mathrm{Ma}$ (Mendoza-Borunda et al., 1998). En la región de Valle Chico una edad similar se documentó para el posible inicio de la deformación al pie de la Sierra San Pedro Martir (Stock y Hodges, 1990) (Figura 4). Lee et al. (1996) proponen que el inicio de la distensión en el sur de Sierra Juárez pudo haber ocurrido desde los $16 \mathrm{Ma}$, mientras que en la región adyacente en Laguna Salada, las edades de exhumación del basamento plutónicometamórfico indican que el inicio de un levantamiento rápido en la Sierra El Mayor ocurrió entre 10 a $15 \mathrm{Ma}$, asociado a una falla de bajo ángulo tipo detachment 


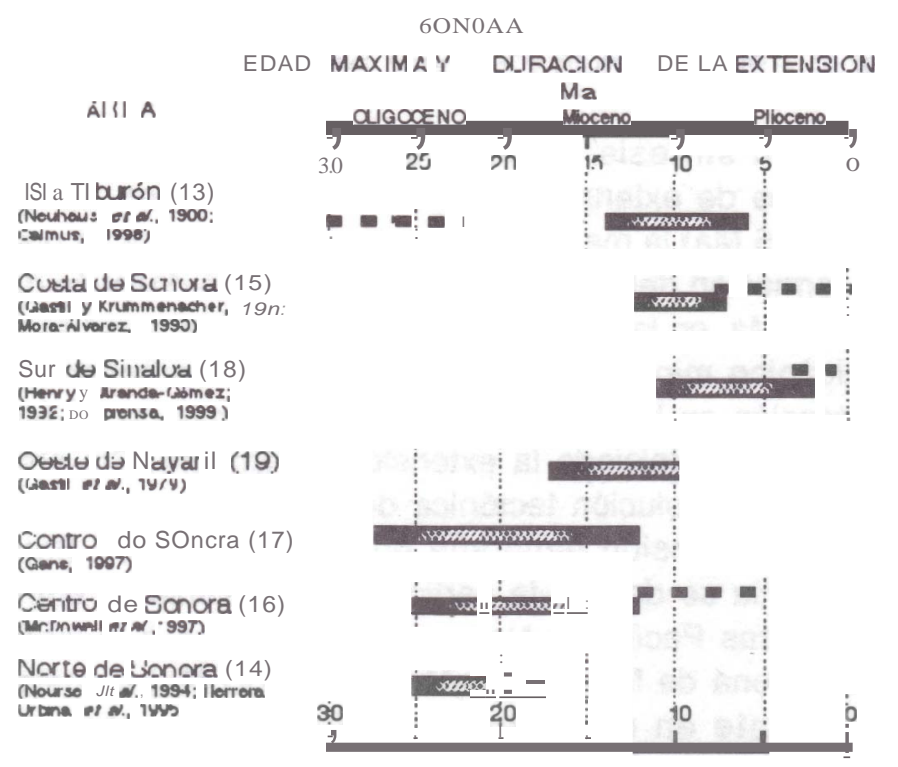

Figura 3. Inicio y duración de la distensión en varias localidades y regiones de Sonora. La barra discontinua indica la edad máxima posible de inicio de la distensión en Isla Tiburón y en Sonora Central (e.g. Calmus eta/., 1998; Herrera-Urbina eta/., 1995)

que corta a depósitos marinos del Plioceno (Siem y Gastil, 1994; Axen et al., en revisión en Tectonics, 1999).

En la región de Bahía de los Ángeles se reportaron sedimentos marinos que posiblemente subyacen a rocas volcánicas fechadas en -14 Ma (Delgado Argote et al., en prensa). Esta última localidad y los depósitos marinos de Isla Tiburón (Neuhaus et al., 1988) son las evidencias más antiguas de'la existencia del protogolfo. Sin embargo, estudios recientes en Isla Tiburón (Mike Oskin, comunicación personal, 1998) cuestionan los resultados que ubican un protogolfo de $>12 \mathrm{Ma}$ y sugieren que la secuencia marina intercalada en rocas volcánicas de Isla Tiburón podría ser más joven «7 Ma). No obstante, en la región de Bahía de los Ángeles la secuencia volcánica terciaria que contiene a los depósitos marinos reportados por Delgado-Argote et al. (en prensa) está coronada por flujos basálticos de -12 Ma con diques alimentadores orientados NNW, lo que sugiere la ocurrencia de extensión temprana en la etapa del protogolfo en esa región (Delgado-Argote, 1999).

El inicio de la distensión en .la región entre San Luis Gonzaga y Bahía de los Angeles está aún pobremente definido entre 15 y -3 Ma. En la región de San Luis Gonzaga, las fallas cortan a flujos de lava de $15 \mathrm{Ma}$, lo que limita la edad máxima del inicio de la extensión en esa región (Martín Barajas et al., en prensa). La ędad mínima está definida pOi' fallas normales que cortan a depósitos marinos pliocénicos?

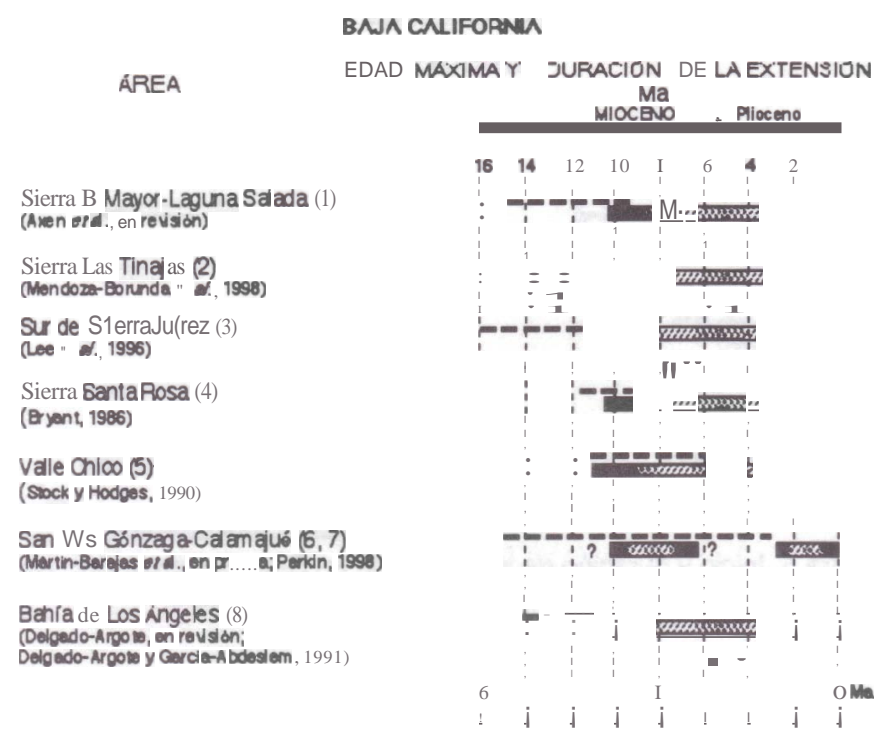

Figura 4. Inicio y duración de la distensión del Mioceno-Plioceno en Baja California. Los números junto a las localidades corresponden a la Figura 1.

que posiblemente se correlacionan con depósitos similares de la región de Puertecitos (Martín-Barajas et al., 1997). Entre Bahía de los Ángeles y San Luis Gonzaga la mayor deformación tectónica en el margen oeste del Canal de Ballenas parece haber ocurrido durante el Plioceno (Parkin, 1998) durante el desarrollo del sistema de falla del Canal de Ballenas que se ha propuesto ocurrió depués de los $3 \mathrm{Ma}$ (c.f. Stock, en prensa).

En Baja California Sur la mayor subsidencia de las cuencas marginales se reporta durante el Plioceno (Figura 5) (Umhoefer et llal., 1994; McLean, 1989; Zanchi, 1994), aunque la cuenca de Santa Rosalía contiene depósitos marinos de hasta $-7 \mathrm{Ma}$ (Holt et aL, en prensa) y la falla San José del Cabo debió tener actividad hace $-11 \mathrm{Ma}$, según estimaciones basadas en el análisis de huellas de fisión y el levantamiento de Sierra la Victoria (Fletcher et al., 1998). Otras evidencias de extensión temprana en la región de La Paz fueron reportadas por Aranda y Pérez-Venzor (1988), aunque Hausback (1984) no consideró que el basculamiento interno de secciones volcanosedimentarias del Grupo Comondú (25 a $12 \mathrm{Ma}$ ) fuera de origen tectónico.

Una característica importante del rift del Golfo de California es su oblicuidad de 15 a $20^{\circ}$ con respecto a la dirección del movimiento relativo de placas Pacífico y Norteamérica. Este ángulo ocasiona una componente de extensión actualmente muý importante en la península de Baja California entre el escarpe principal del Golfo y el sistema de fallas transformes y centros 


\section{BAJA CAUFORNIA SUR}

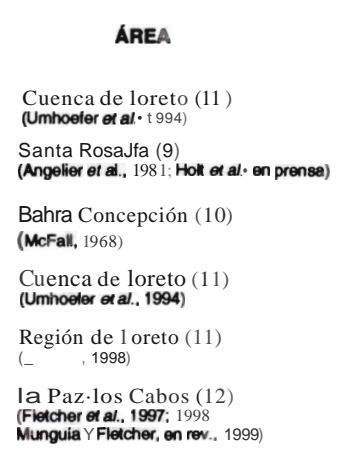

EDAD MÁXIMA Y DURACIÓN DE LA EXTENSIÓN Ma
MIOCENO I PLIOCENO
$j$ $\begin{array}{lllllllll}16 & 14 & 12 & 10 & 8 & 6 & 4 & 2 & 0\end{array}$ $-\frac{1}{-\infty}$
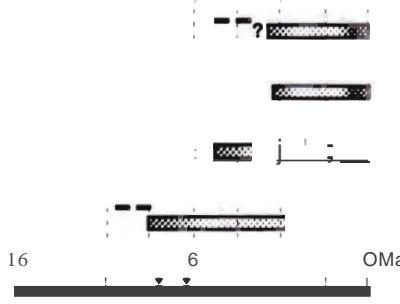

Figura 5. Inicio y duración de la distensión en Baja California Sur. Los números junto a las localidades corresponden a la Figura 1.

de dispersión del Golfo (Stock et al., 1996; Axen y Fletcher, 1998; Umhoefer y Dorsey, 1997). Varios autores han aportado evidencias de un cambio de orientación de esfuerzos durante la evolución del protogolfo al golfo moderno (c.f. Angelier et al.,1981; Zanchi, 1994), y es ampliamente aceptado que el Golfo de California se inició como un rift en el cual predominó una componente de extensión E-NE perpendicular a la orientación de las márgenes del rift, con la deformación transpresiva acomodada primero en la zona de falla San Benito-Tosco-Abreojos (Figura 2) y posteriormente en las fallas transformes del Golfo (Stock y Hodges, 1989). Un cambio hacia el NW en la dirección de movimiento relativo de la placa Pacífico (Atwater y Stock, 1998) ocasionó un ángulo de 20 a $30^{\circ}$ entre la orientación del rift y la dirección de movimiento relativo de la placa del Pacífico. La evolución a un rift oblicuo con la captura de la península por la placa del Pacífico hacia los $5 \mathrm{Ma}$ conlleva una componente de extensión que se manifiestó en la península con la subsidencia de cuencas marginales (Stock et al., 1996; Axen y Fletcher, 1998; Lonsdale, 1989).

En contraste con la costa de Sonora, la componente extensional del sistema transforme San Andrés-Golfo de California es aún importante en la península de Baja California y controla la subsidencia de las cuencas adyacentes al escarpe de Sierra Juárez y Sierra San Pedro Martir (Dokka y Merriam, 1982; Stock y Hodges, 1989; Stock et al., 1996; Axen y Fletcher, 1998).

En Baja California Sur la componente de extensión de este rift oblicuo se manifiesta en la cuenca de Loreto de edad Plioceno (Umhoefer y Dorsey, 1997; Zanchi, 1994), y en la cuenca de San José de los Planes del Mie-Plioceno (Fletcher el al., 1997). La falla maestra en el borde occidental de esta cuenca corta al aluvión cuaternario y se considera aún activa (Fletcher et al., 1997; Munguía y Fletcher, en revisión,Tectonics).

En síntesis, aunque en la península existen reportes de extensión temprana en el Mioceno medio (ca. - $15 \mathrm{Ma}$ ) la mayor parte de los estudios indican que la formación del escarpe del Golfo se inició después de los $11 \mathrm{Ma}$, en la posición que ocupaba el frente del arco volcánico miocénico recién extinto. Este proceso de extensión en la península se manifestó más de $15 \mathrm{Ma}$ después de iniciada la extensión en Sonora. Sin embargo, la evolución tectónica de rifts oblicuos sugiere que la extensión temprana en la península de Baja California se debe a la partición del movimiento entre las placas Pacífico y Norteamérica, primero a lo largo de la zona de falla Tosco-Abreojos (12-5 Ma) y posteriormente en el sistema transforme y centros de dispersión del Golfo (e.g., Stock y Hodges, 1989). El ángulo de más de $20^{\circ}$ entre la orientación del rift y el azimut del movimiento relativo de la península genera un componente de extensión y con fallamiento normal y de rumbo en la península (Stock y Hodges, 1989; Suárez-Vidal et al., 1991; Axen y Fletcher, 1998, Umhoefer y Dorsey, 1997; Munguía y Fletcher, en revisión). Las variaciones estructurales a lo largo del margen de la península posiblemente se deben a rasgos estructurales heredados durante la distensión del protogolfo. En el lado opuesto del Golfo la sedimentación ha enmascarado las estructuras miopliocénicas y no se tienen reportes de fallas activas y/o cuaternarias en la franja costera de Sonora y Sinaloa. El alineamiento N-S de conos cineríticos en el campo volcánico El Pinacate (Lynch, 1981) Y la actividad de la falla Pitaycachi en el noreste de Sonora son las únicas evidencias de deformación activa en Sonora (Suter, 1991).

\section{EVOLUCION MAGMÁTICA}

El volcanismo neogénico en la Provincia Extensional del Golfo muestra una evolución en el estilo eruptivo y en su composición química, que refleja el cambio del régimen de subducción al desarrollo de un rift continental, en una región previamente extendida durante el proceso de extensión de la provincia de Cuencas y Sierras (Basin and Range) (c.f., Henry y Aranda-Gómez, 1992). Entre - 12 y 5 Ma la zona occidental de esta zona de extensión evolucionó a un rift oceánico, con la transferencia progresiva de la penín-sula de Baja California a la placa del Pacífico.

Al término de la subducción (16-12 Ma) se registra un cambio en el estilo y la composición del volcan ismo en la Provincia Extensional del Golfo. En varias 


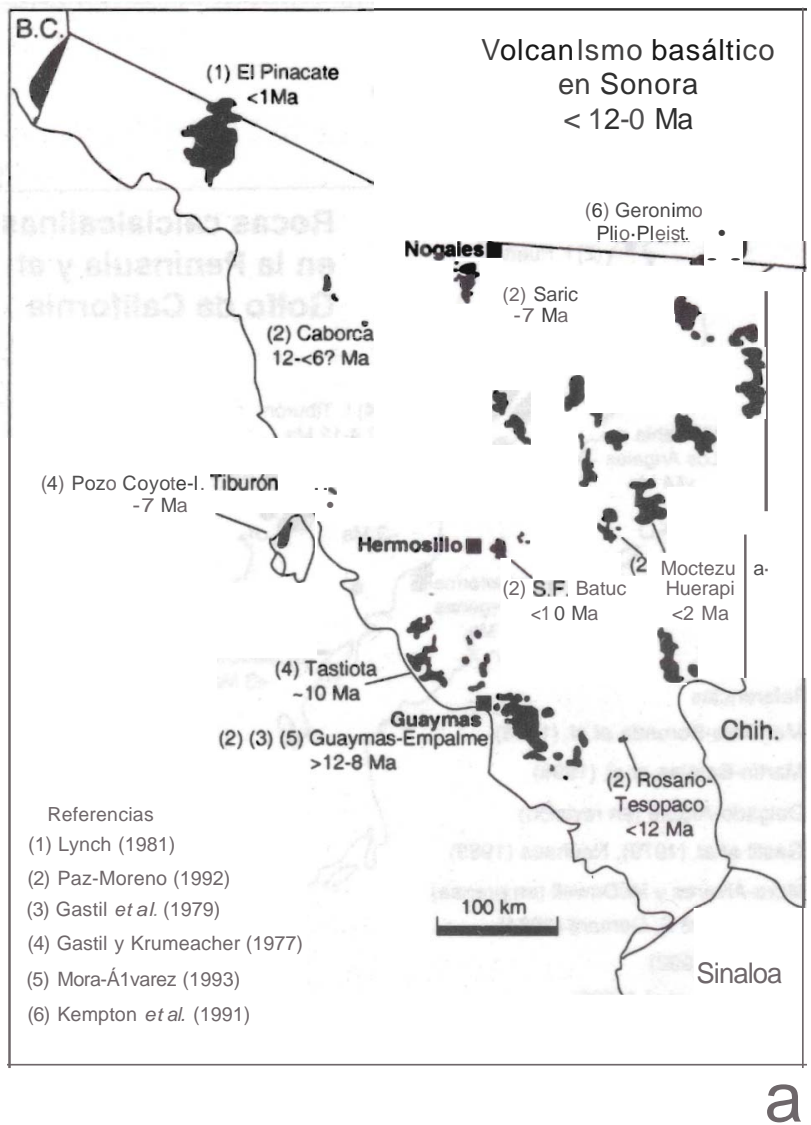

Figura 6a. Distribución del volcanismo basáltico y andesítico de edad Mio-Plio-Cuaternario en Sonora y la península de Baja California. modificado de la Carta Geológica de la República Mexicana escala 1:2 000000 (Ortega-Gutiérrez et al., 1992). Las localidades con volcanismo basáltico en Sonora son de Paz-Moreno (1992).

regiones de la península se ha documentado que depósitos de ignimbritas de 14-10 Ma y flujos de lava andesítico-basáltica de -12-8 Ma sobreyacen a la secuencia volcanosedimentaria del arco miocénico de composición principalmente dacítica-andesítica. El cambio a un volcanismo bimodal riolítico-basáltico está acompañado de una diversificación geoquímica importante de lavas alcalinas, toleíticas y calcialcalinas. Los factores que controlan la composición de la fuente y los patrones de evolución magmática aún no han sido explicados suficientemente, debido a la falta de análisis isotópicos en muchas de las localidades. Sin embargo, se reconocen algunas tendencias generales a través de la Provincia Extensional del Golfo.

A escala regional, el volcanismo asociado a la evolución del rift del Golfo de California presenta un patrón geográfico en el cual predominan lavas alcalinas en los extremos de esta provincia, tanto en Sonora como en la porción estable de la península (Figura 6a y 6b). En Baja California Sur, flujos de lava andesítica y basáltica de afinidad alcalina fueron emplazados hacia el poniente del escarpe principal del Golfo durante

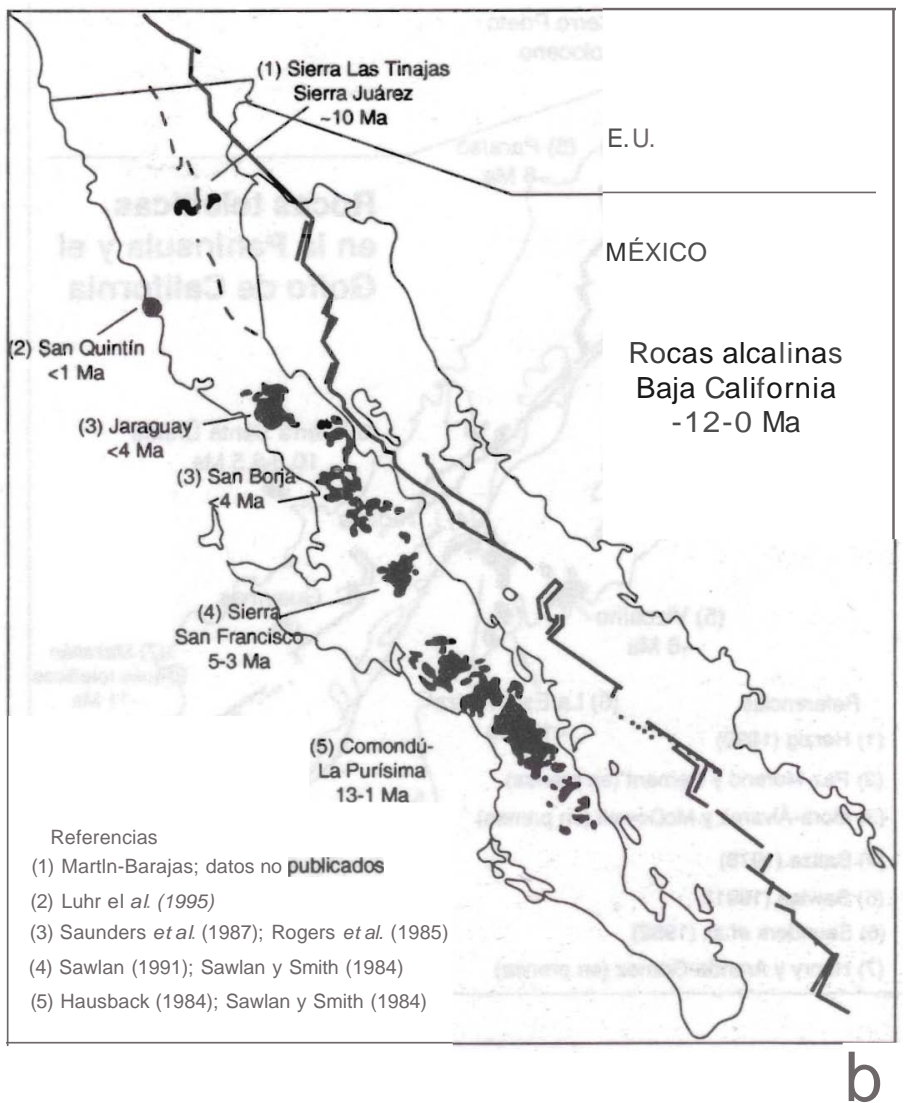

Figura 6b. Distribución de afloramientos con volcanismo basáltico alcalino del Neógeno y Cuaternario en la península de Baja California.

13 Y $1 \mathrm{Ma}$ (Sawlan, 1991) (Figura 6b). En los campos volcánicos de Jaraguay y San Borja del norte de la península, se reportaron andesitas de 4 a OMa de edad con alto contenido en $\mathrm{MgO}, \mathrm{Ni}, \mathrm{Cr}$ y Sr. (Saunders et al.,1987; Rogers et al., 1985). Estos autores argumentaron que esas características corresponden a magmas derivados de la placa oceánica y la dorsal subducidas. Sin embargo, Sawlan (1991) atribuyó estas caracterís-ticas a la fusión parcial en un manto metasomatizado por fluidos durante la subducción.

Otras localidades con volcanismo andesítico basáltico alcalino se han reportado en los últimos años en la depresión del Golfo. Los flujos traquibasálticos de $c a$. $10 \mathrm{Ma}$ que coronan la secuencia volcánica en el sur de Sierra Las Tinajas, indican magmas poco evolucionados y enriquecidos en elementos incompatibles (Martín-Barajas, datos no publicados). A pesar de su afinidad alcalina, estos flujos 'también presentan características geoquímicas heredadas del volcanismo calcialcalino de subducción, como el empobrecimiento de $\mathrm{Nb}$, Ta y $\mathrm{Ti}$ con respecto a los basaltos de piso oceánico (MORB). La contaminación de los magmas por fusión de la corteza aparece como 


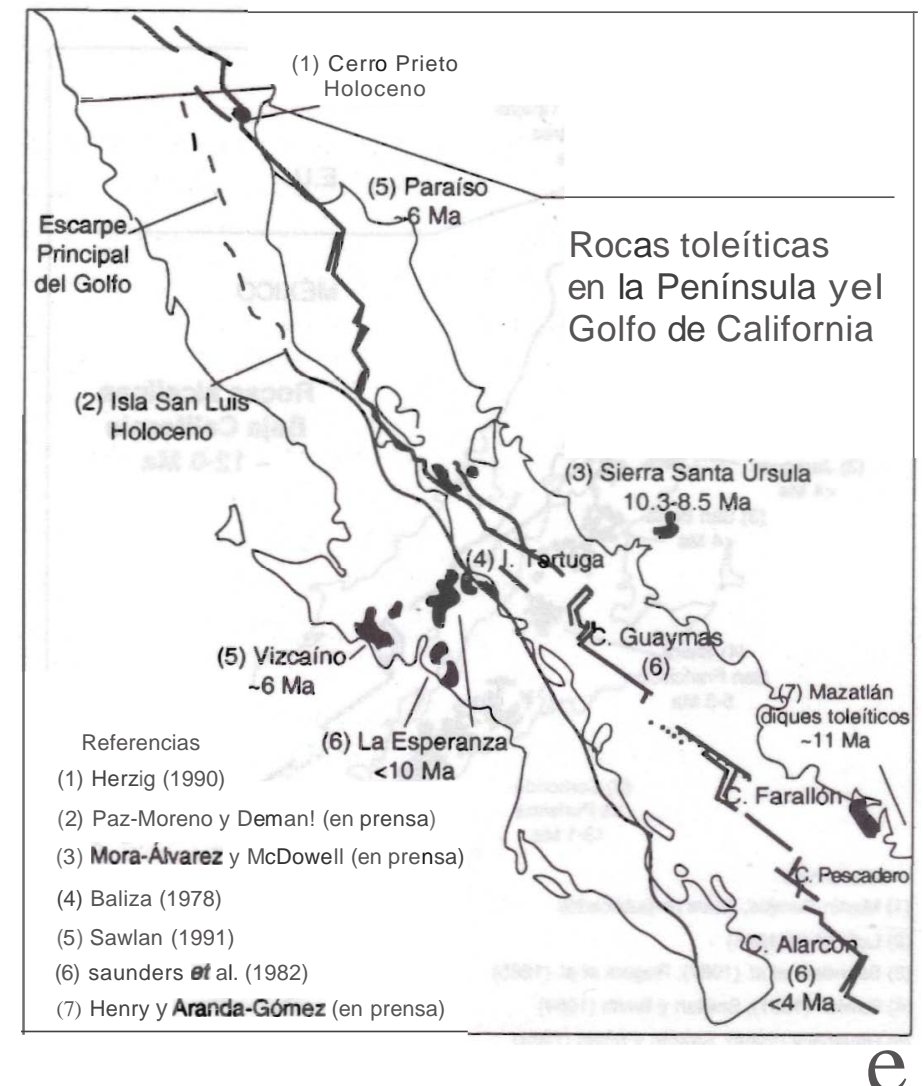

Figura 6c. Volcanismo toleítico del Neogeno y Cuaternario en la península de Baja California y el Golfo.

una constante en varias de la localidades estUdiadas, aunque solo en pocos de ellos se han realizados modelos de evolución magmática que permitan estimar la contribución de la contaminación cortical.

En la región central de Sonora el volcanismo basáltico alcalino posterior a la subdución se inicia hacia los $10 \mathrm{Ma}$ (Paz-Moreno, 1992). El volcanismo alcalino se presenta en varios campos discretos de edad Mio-Plio-Cuaternario (Figura 6a) (Paz-Moreno, 1992). Son basaltos y andesitas microporfiríticos de plagioclasa, piroxeno y olivino, enriquecidos en elementos incompatibles, incluyendo $\mathrm{Nb}$, por lo que se ha sugerido que provienen de magmas generados en el manto litosférico (Paz-Moreno, 1992). El campo volcánico El Pinacate, en la parte noroccidental y norte de Sonora, es un ejemplo de magmatismo alcalino PlioCuaternario, con posible actividad holocénica (Lynch, 1981). Está compuesto por basaltos alcalinos (basanitas) y sus productos diferenciados (traquitas). El volca-nismo más joven en el Pinacate incluye también toleítas (Lynch, 1981). Con base en estudios isotópicos se ha sugerido que este campo volcánico está asociado a un manto astenosférico, aunque su relación con la apertura del Golfo de California aún es incierta (Lynch

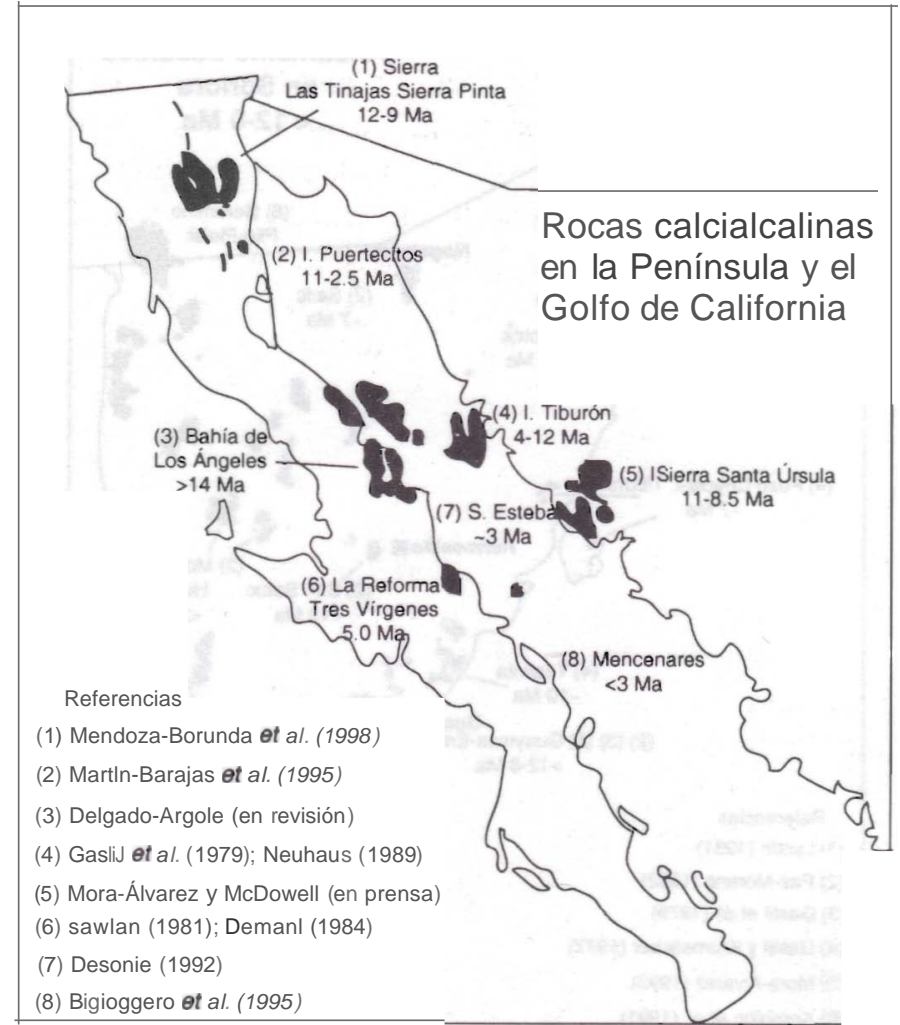

Figura 6d. Volcanismo calcialcalino del Neogeno y Cuaternario en la Provincia Extensional del Golfo.

et al., 1993), ya que está ubicado sobre corteza continental y a $50 \mathrm{~km}$ al este de centro de dispersión de Cerro Prieto.

El volcanismo toleítico (10-0 Ma) se presenta predominantemente en las márgenes y en los centros de dispersión del Golfo de California (Figura 6c) (Saunders, 1982; Batiza et al., 1979, Herzig, 1990; PazMoreno y Oemant, 1999; Mora-Álvarez y McOowell, en prensa). Sin embargo, flujos de lava de afinidad toleítica del Mioceno tardío tambien se presentan al oeste del escarpe del Golfo en la península (Figura 6c). En Baja California Sur, los basaltos Esperanza $(-10 \mathrm{Ma})$ y flujos andesíticos de la península de Vizcaíno (-6 Ma) indican magmas toleíticos fueron emplazados en una región al parecer no afectada por la distensión en la etapa del protogolfo. Este volcanismo toleítico transicional (c.f., Sawlan, 1991) presenta enriquecimiento en elementos incompatibles, por lo que se ha sugerido que provienen de la fusión selectiva de venas de piroxenita hospedadas en rocas más refractarias del manto, de composición peridotítica (Sawlan, 1991 ).

En la costa de Sonora, el volcanismo basáltico toleítico en la etapa del protogolfo se ha reportado en la 
región de Empalme con edades de 8 a 10 Ma (MoraÁlvarez, 1993). Diques basálticos toleíticos de ca.10 Ma y emplazados en estructuras con orientación NNW han sido estudiados en la costa de Sinaloa (Henry y Aranda-Gómez, en prensa). Estas manifestaciones volcánicas, junto con las toleítas transicionales de Baja California (Sawlan, 1991) representan el ascenso de un manto astenosférico en la etapa del protogolfo, que se manifestó dentro de la zona de distensión y en la zona estable de la península, al parecer no afectada por la extensión de la corteza.

El volcanismo calcialcalino en la Provincia Extensional del Golfo es principalmente dacítico-riolítico y ocurre en campos volcánicos discretos en las márgenes y en algunas islas. La provincia volcánica de Puertecitos es posiblemente la más grande en la península.con volcanismo riolítico-dacítico calcialcalino (Stock, 1989; Martín-Barajas et al., 1995) (Figura 6d). Se han documentado tres períodos de actividad de tipo caldérica que ocurrieron hacia $-11,6$ Y $3 \mathrm{Ma}$, respectivamente. En la región de Loreto, el campo volcánico de Mencenares está formado principalmente de domos y flujos dacíticos con afinidad calcialcalina de edad Plio-Cuaternario (Bigioggero et al., 1995). Algunas islas del Golfo (e.g., San Esteban, San Lorenzo y Ángel de la Guarda) contienen secuencias volcánicas que corresponden a una actividad efusivaexplosiva del Mioceno tardío-Plioceno, esencialmente calcialcalina y con magmas diferenciados (Desonie, 1992; Escalona-Alcazar, 1999). En la región de Santa Rosalía, la caldera La Reforma (Plioceno) y el complejo volcánico de Tres Vírgenes (Plio-Cuaternario) están compuestos por volcanismo andesítico-dacítico dominante y de afinidad calcialcalina (Sawlan, 1981), aunque Demant (1981) tambien reporta lavas comendíticas en la caldera La Reforma.

Se desconoce si el volcanismo riolítico-andesítico calcialcalino es producto de la diferenciación de magmas basálticos generados por fusión parcial en el manto o si se trata de magmas generados por la fusión de la corteza, o de una combinación de estos dos procesos. Este tipo de volcanismo conserva muchas de las características geoquímicas del volcanismo de arco y su variación posiblemente representa diversos grados de contaminación cortical.

\section{DISCUSiÓN}

La evolución del volcanismo neogénico y ei proceso distensivo del noroeste de México estan enmarcados en dos eventos tectónicos regionales relacionados. Ël primero es el encuentro de la dorsal
Pacífico-Farallón con la trinchera de Norteamérica hacia los $29 \mathrm{Ma}$ que ocasionó la terminación progresiva del volcanismo de arco junto con la migración hacia el sur de la junta triple (dorsal-trinchera-falla transformante) (Atwater, 1989; Stock y Lee, 1994). La zona de fractura San Benito-Tosco-Abreojos funcionó como una falla transformante a lo largo de la margen occidental de la península y constituyó el límite de placas entre 12 y -5 Ma (Spencer y Normak, 1989; Lonsdale, 1989). El segundo evento es el cambio en el límite de placas hacia el Golfo de California y la transferencia progresiva de la península a la placa Pacífico. Durante el período entre 12 y $4 \mathrm{Ma}$ la península mantuvo un movimiento relativo independiente funcionando como una microplaca; la transferencia de la península a la Placa Pacífico se completó hacia los $4 \mathrm{Ma}$, como sugieren las anomalias magnéticas más antiguas (3.5 Ma) en la boca del Golfo (Lonsdale, 1989; Stock y Hodges, 1989).

La subsidencia inicial de las cuencas en la etapa del protogolfo fue posiblemente dirigida por una tectónica distensiva de dirección E-NE que prevaleció durante la etapa pre-5 Ma (e.g., Angelier et al., 1981). Las únicas evidencias se han definido en las márgenes del Golfo, principalmente en Baja California, y recientemente en el sur de Sinaloa (Henry y ArandaGómez, en prensa). En el norte de la penísula, las fallas maestras que controlan la subsidencia de las cuencas mio-pliocénicas cambian de polaridad del sentido de transporte de la placa superior (c.f. Axen; 1995). La segmentación en la etapa temprana del rift en segmentos estructurales de 50 a $100 \mathrm{~km}$, separados por zonas de acomodamiento, corresponde a una característica común en otros rifts continentales de tipo ortogonal (e.g., Bosworth, 1985), y las cuencas adyacentes al escarpe del Golfo y en la costa de Sonora-Sinaloa pudieron haberse formado de esta manera. Algunas de las fallas originadas durante la etapa temprana posiblemente permaneciendo activas con el cambio a un rift oblicuo, acomodando la componente del extensión en la península.

Bajo el continente, el desarrollo de una ventana astenosférica durante el Neógeno producida por la subducción de la dorsal y el jalón gravitacional del slab o corteza oceánica subducida, debió modificar la estructura y las características térmicas de la litosfera en una amplia zona del suroeste de Estados Unidos y del noroeste de México (c.f., Severinghaus y Atwater, 1990; Dickinson, 1997). Esta hipótesis ha sido discutida en los últimos años y parece haber ganado muchos adeptos entre la comunidad de geociencias. Es posible que el origen de la extensión y el magmatismo intermedio a félsico en el suroeste de Arizona ha sido 
atribuido al colapso gravitacional que siguió a la orogenia Laramide (Spencer et al., 1995), sin embargo, este proceso difícilmente puede explicar las firmas geoquímicas de tipo OIB (Oceanic Island Basalts) que predominan en el volcanismo basáltico plio-cuaternario en algunas regiones del suroeste de Estados Unidos, en Sonora y el norte de Baja California, el cual requiere de la fusión de un manto astenosférico y menor participación del manto litosférico (e.g., Fitton et al., 1991; Paz-Moreno, 1992).

Las relaciones entre causa y efecto aún no logran consenso debido a que en muchas áreas estudiadas el volcanismo precede a la extensión y en otras el proceso distensivo antecede o es contemporáneo al volcanismo (ver síntesis en Metcalf y Smith, 1995). En Sonora el proceso de extensión temprana concuerda con una reactivación del volcanismo, principalmente de composición basáltica, que precedió a la actividad de la Sierra Madre Occidental (e.g., McDowell el al., 1997). Las lavas basálticas intercaladas en los depósitos continentales de la Formación Baucarit en Sonora central sugieren que el magmatismo y la extensión están íntimamente ligados, y se ha propuesto que el magmatismo máfico del Mioceno temprano pudo haber debilitado térmicamente la litosfera y ocasionar la extensión de la corteza (Gans, 1997). No obstante, este tipo de volcanismo se ubica en una posición trasarco y aún se desconoce cual es su relación genética con la subducción de la Placa Farallón-Guadalupe al oeste de la península.

Para explicar la variación espacial y temporal del volcanismo y su composición es necesario considerar las características geoquímicas de la fuente de los magmas. Desafortunadamente, son pocos los estudios isotópicos en lavas andesítico-basálticas y en xenolitos de la base de la corteza y del manto, por lo que tal variación de la fuente y los patrones de evolución magmática aún no pueden definirse. Sin embargo, podemos suponer que la variación en las características geoquímicas del volcanismo máfico si refleja una heterogeneidad de la fuente o diferentes grados de fusión parcial. La evolución de estos magmas en la corteza, la ubicación de los centros de emisión y el estilo eruptivo del volcanismo post-subducción «12 Ma) están, en la mayoría de los casos, fuertemente controlados por los patrones estructurales.

El análisis de xenolitos ultramáficos en varias localidades del centro y occidente de México sugiere que las presiones y temperaturas de equilibrio entre los xenolitos y las lavas que los contienen corresponden a un proceso de fusión parcial somera $(-25 \mathrm{~km})$ y una corteza más delgada en la región del Golfo y en el margen Pacífico (Luhr y Aranda-Gómez, 1997). El estudio de Luhr y Aranda-Gómez (1997) concluye que el manto litosférico bajo el campo volcánico de San Quintín también muestra la influencia de fluidos derivados de la antigua subducción. Aunque aún no es claro si esa actividad volcánica, la única con volcanismo basáltico intraplaca en la península, está controlada por estructuras relacionadas a la distensión regional.

Nuestro conocimiento sobre el origen y la evolución del magmatismo neogénico en el noroeste de México se ha ampliado a medida que se publican los estudios de campo y estudios petrogenéticos del volcanismo asociado a la distensión de la corteza. Estos estudios permiten acotar los modelos geofísicos sobre la estructura de la corteza en esta geológicamente compleja región, cuyos procesos tienen gran impacto en la sociedad por sus recursos naturales y por el riesgo geológico inherente a las actividades sísmica y volcánica de esta porción del noroeste de México.

\section{REFERENCIAS BIBLIOGRÁFICAS}

Angelier, J.; Colleta, B.; Chorowicz, J.; Ortlieb, L. y Rangin, C., 1981, Fault tectonics of the Baja California Peninsula and the opening of the Sea of Cortez, Mexico: Journal of Structural Geology, v. 3, p. 347-357.

Atwater, T., 1989, Plate tectonic history of the Northeast Pacific, in D. Winterer, M. Hussong, and D. R.W., oos., The Eastern Pacific and Hawaii, Boulder, CO, Geological Society of America, Geology of North America, v. N, p. 21-73.

Atwater, T, y Stock, J. M., 1998, Pacific-North Amerlca piate tectonics of the Neogene southwestern United States: An update: International Geology Review, v. 40, p. 375-402.

Aranda-Gómez, J., y Pérez-Venzor, J. A., 1988, Estudio geológico de Punta Coyotes, Baja California Sur: Universidad Nacional Autonoma de Mexico, Instituto de Geología, Revista, v. 7, p. 1-21.

Axen, G., 1995, Extensional Segmentation of the Main Gulf Escarpment, Mexico and the United States: Geology, v. 23, p.515-518.

Axen, G. J., y Fletcher, M. J.,1998, Late Miocene-Pleistocene extensional faulting, northern Gulf of California, Mexico and Salton Trough, California: International Geology Review, v. 40, p. 217-244.

Axen, G. J.; Grove, M.; Stockli, D.; Lovera, O. M.; Rothstein, D. A.; Fletcher, M. J.; Farley, K. y Abbott, L. P., Thermal evolution of Monte Blanco dome-late Neogene low-angle normal faulting during the Gull of California rifting and late Eocene disruption of extraregional river systems: enviado a Tectonics, abril 1999.

Batiza, R.; Futa, K.y Hedge, C. E., 1979, Trace elements and strontium isotope characteristics of volcanic rocks from Isla Tortuga- a young seamount in the Gulf of California: Earth and Planetary SCience Letters, v. 43, p. 269-278.

Bigioggero, B.; Chiesa, S.; Zanchi, A.; Montrasio, A. y Vezzoli, L., 1995, Post-subduction magmatism within the Gulf of California Rift: The Cerro Mencenares Volcanic Center, Baja California Sur, Mexico: Geological Society of America Bulletin, v. 107, p. 1,108-1,122.

Bosworth, W., 1985, Geometry of propagating continental rifts: Nature, v. 316, p. $625-627$. 
Bryant, B., 1986, Geology 01 the Sierra Santa Rosa basin, Baja Calilomia, Mexico [Master thesis): San Diego State University, $75 \mathrm{p}$.

Calmus, T.; Poupeau, G.; Delaux, T. y Labrin, T., 1998, Apatite lission track ages in Sonora, Mexico: a recording 01 Basin and Range events and opening 01 the Gull 01 Calilornia, Puerto Vallarta, Jalisco, Reunión anual de la Unión Geolísica Mexicana, Geos, v. 18, n. 4, p. 293 (abstract).

Delgado-Argote, L. A.; López-Martinez, M. y Perrillat, M. C. en prensa, Geologic reconnaissance and age 01 volcanism and associatoo launa Irom sediments 01 Bahia de los Angeles, central Gull 01 Calilornia, in Delgado, H. Stock, J. M. Y Aguirre, G. eds., Cenozoic tectonics and volcanism in Mexico: Boulder CO., Geological Society 01 America Special Paper 334, en prensa.

Delgado-Argote, L.A. y García-Abdeslem, J., 1999, Shallow Miocene basaltic magma reservoirs in the Bahia de los Angeles Basin, Baja Calilornia, Mexico, Journal 01 Volcanology and Geothermal Research, v. 88-1, p. 29-46

Delgado-Argote, L. A., 1999, Evolución tectónica y magmatismo Neógeno de la margen oriental de Baja Calilornia central. Tesis de Doctorado: Instituto de Geologia, Universidad Nacional Autonoma de Mexico, en trámite, septiembre, 1999.

Demant, A., 1981, Plio-Ouaternary volcanism 01 the Santa Rosalia area, Baja Calilornia, Mexico, in Orlieb, L. y Roldan-O, J., OOs., Geology 01 northwestern Mexico and southern Arizona, lield guides and papers: Hermosillo, Sonora: Geological Society 01 America, Cordilleran Section, Annual Meeting, p. 295-305.

Desonie, D. L., 1992, Geologic and geochemical reconnaissance 01 Isla San Esteban-post-subduction orogenic volcanism in the Gulf 01 Calilornia: Journal 01 Volcanology and Geothermal Research, v. 52, n. 1-3, p. 123-140.

Dickinson, W. R., 1997, Tectonic implications 01 Cenozoic volcanism in coastal Calilornia: Geological Society 01 America Bulletin, v. 109, p. 936-954

Dokka, R. K.y Merriam, R. H., 1982, Late Cenozoic extension 01 northwestern Baja Calilornia, Mexico: Geological Society 01 America Bulletin, v. 93, n. 5, p. 371-378.

Escalona Alcázar, F. J., 1999, Reconocimiento geológico de la costa occidental de la isla San Lorenzo y Bahía de las Animas.Tesis de Maestría: Ensenada, Baja Calilornia, Centro de Investigacion Cientilica y Educación Superior de Ensenada, en tramite, septiembre, 1999.

Ferrari, L.; López-Martínei, M.; Aguirre-Diaz, G. y Carrasco-Núñez, G., 1999, Space-time patterns 01 Cenozoic arc volcanism in central Mexico- Irom the Sierra Madre Occidental to the Mexican Volcanic Belt: Geology, v. 27, p. 303-306.

Fitton, J. G.; James, D. y Leeman, W. P., 1991, Basic magmatism associatOO with Late Cenozoic extension in the western UnitOO States: Compositional variations in space and time: Journal 01 Geophysical Research, v. 96, p. 13,693-13,711.

Fletcher, M. J.; Foster, D.; Kohn, B. y Ramos-Velázquez, E.,1998, Differential cooling and uplift 01 the crystalline basement in the La Paz-Los Cabos region, Baja Calilornia Sur, Mexico: Geological Society 01 America, Abstracts with Programs, v. 30, p.14.

Fletcher, M. J., Munguía-Orozco, L., Pérez-Venzor, J. A., Foster, D. y Aranda-Gomez, J.,1997, Transtensional Tectonics, Southern Gull 01 Calilornia: EOS, Transactions, American Geophysical Union, v. 78, p. F822.

Gans, P. B., 1997, Large-magnitude Oligo-Miocene extension in southern Sonora-Implications lor the tectonic evolution 01 northwest Mexico: Tectonics., v. 16, p. 388-408.

Gastil, G., and Krummenacher, D., 1977, Reconnaissance geology 01 coastal Son'ora between Puerto Lobos and Bahia Kino.: Geological Society 01 America Bulletin, v. 88, p. 189-198.

Gastil, G., Krummenacher, D., and Minch, J., 1979, The record 01 Cenozoic voicanism around the Gulf 01 Calilornia.: Geological
Society 01 America Bulletin, v. 90, p. 839-857.

Hausback, B. P., 1984, Cenozoic volcanic and tectonic evolution of Baja Calilomia Sur, Mexico, in Frizzell, V. A., oo., Geology 01 the Baja Calilornia Peninsula: Tulsa OK, Society 01 Economic Paleontologists and Mineralogists, Pacific Section, p. 219-236.

Henry, C. D., y Aranda-Gómez, J. J., 1992, The real southem Basin and Range: Mid- to late Cenozoic extension in Mexico: Geology, v. 20, p. 701-704.

Henry, C. D., and J. Aranda-G6mez, Plate interaetions control MiddleLate Miocene, proto-Gulf and Basin and Range Extension in the southem Basin and Range: Tectonophysics, en prensa.

Herrera-Urbina, S.; Ortega-Rivera, A.; Farrar, E., y Cocheme, J., 1995 , Some apparent ${ }^{40} \mathrm{Ar} /{ }^{39} \mathrm{Ar}$ ages lor metamorphic rocks in north central Sonora, Mexico and their interpretation: Boletín del Departamento de Geología, Universidad de Sonora, v. 12, p.85-100.

Herzig, T. C., 1990, Geochemistry 01 igneous rocks from the Cerro Prieto geothermal lield, northern Baja Calilornia, Mexico: Journal 01 Volcanology and Geothermal Research, v. 42, p.261-271

Holt, J. W.; Holt, E. W. y Stock, J. M., An age constraint on Gulf 01 Calilomia rifting Irom the santa Rosalia basin, Baja Califomia Sur, 01 earliest marine sedimentary lormation at santa Resalía, Baja Calilornia Sur, Mexico: Geological Society 01 America Bulletin, en prensa.

Kempton, P. D., Fitton, J. G., Hawkesworth, C. J., and Ormerod, O. S., 1991, Isotopic and trace elements on the composition and evolution 01 the litosphere beneath the southwestem Unitoo States: Journal 01 Geophysical Research, v. 96, no. B6, p.13,713-13,735.

Lee, J.; Miller, M. M.; Crippen, R.; Hacker, B. y LOOesma-Vázquez, J., 1996, Middle Miocene extension in the Gulf Extensional Province, Baja Calilornia-Evidence Irom the southem Sierra Juarez: Geological Society 01 America Bulletin, v. 108, p.505-525.

Lonsdale, P., 1989, Geology and tectonic history 01 the Gulf of Califomia, in Winterer, D., Hussong, M y Decker, R.W., OOs., The eastern Pacilic and Hawaii, Geological Society of Amenea, Boulder, Co., The Geology of North Amenea, v. N, p. 499-521.

Luhr, J. F., Aranda-Gomez, J. J., Y Podosek, F. K., 1995. The San Ouintín volcanic lield, Baja Califomia Norte, Mexico-geology, petrology, and geochemestry: Journal 01 Geophysical Research, v. 100, p. 10,353-10,380.

Luhr, J. F. Y Aranda-Gómez, J. J., 1997, Mexican peridotite xenoliths and tectonic terranes: correlations among vent location, textura, temperature, pressure, and oxygen lugacity: Journal 01 Petrology, v. 38, n. 8, p. 1,075-1,112.

Lynch, O.J., 1981, Genesis and petrology 01 alkaline volcanism in the Pinacate volcanic lield 01 northewestern Sonora, Mexico: Tuczon Arizona, University 01 Arizona, Ph.D. Thesis, 251 p.

Lynch, D. J.; Musselman, T. E.; Gutmann, J. T. YPatchett, P. J., 1993, Isotopic evidence lor the origin 01 Cenozoic volcanic rocks in the Pinacate volcanic lield, northwestem Mexico: Lithos, v. 29, p.295-302

Martín-Barajas, A., Stock, J. M.; Layer, P.; Hausback, B.; Renne, P. y López-Martínez, M., 1995, Arc-rift transition volcanism in the Puertecitos Volcanic Province, northeastem Baja Calilornia, Mexico: Geological Society 01 America Bulletin, v. 107, n. 4, p.407-424.

Martín-Barajas, A., Téllez-Duarte, M., y Stock, J. M., 1997, The Puertecitos Formation: Pliocene volcaniclastic sOOimentation along an accommodation zone in northeastem Baja California, in Johnson, M. E. and LOOesma-Vázquez, J., OOs., Pliocene Carbonate and RelatOO Facies Flanking the Gulf 01 Califomia, Baja Calilomia, Mexico, Boulder Colorado, Geological Society 01 America, Special Paper 318, p. 1-24.

Martín-Barajas, A.; Fletcher, M. J.; López-Martínez, M. y Mendoza- 
Borunda, $\mathrm{R}$, Waning Miocene subduction and arc volcanism in Baja Califomia: The San Luis Gónzaga volcanic field, in Ferrari, L.; Stock, J. M. and Urrutia-Fucugauchi, J. OOs., Post-Laramide magmatism and tectonics in Mexico and plate interaction: Tectonophysics, special issue, en prensa.

McDowell, F. W.; Roldán-Quintana, J. y Amaya-Martínez, R., 1997, The interrelationship of sedimentary and volcanic deposits associated with tertiary extension in Sonora, Mexico: Geological Society of America Bulletin, v. 109, n. 10, p. $1,349-1,360$.

McFall, C. C., 1968, Reconnaissance geology 01 the Concepcion Bay area, Baja Califomia, Mexico: Stanford University Publications in Geological Sciences, v. 10, p. 115-122.

McLean, H., 1989, Reconnaissance geology of a Pliocene marine embayment near Loreto, Baja Califomia Sur, Mexico: Pacific Section Society of Economic Paleontologists and Mineralogists, Field Trip Guidebook, v. 63, R. 17-25.

Mendoza-Borunda, Ramón; Axen, Gary; Sandeman, Hamich; Amabal Ortega-Rivera y Grover, Tracy M., 1998, Stratigraphy and geochronology of the Tertiary volcanic and sOOimentary rocks in the southern Sierra Juarez and northem Sierra Las Tinajas area, in northeastern Baja California, Mexico: Puerto Vallarta Jalisco, Reunion Anual de la Unión Geofísica Mexicana: Geos, v. 18, n. 4, p. 292-293 (abstract).

Metealf, RV. and Smith, E.I., 1995, Introduction to special section: Magmatism and extension: Joumal 01 Geophysícal Research, v. 100 , p. $10,249-10,253$.

Mora-Á1varez, G., 1993, Relaciones estratigráficas y geocronólogicas entre las unidades volcánicas de la Sierra Santa Úrsula en Sonora, y el magmatismo de la región del Golfo de California, in Oelgado-Argote, L, y Martín-Barajas, A., eds., Contribuciones a la Tectónica del Occidente de México, Ensenada, Baja California, Unión Geofísica Mexicana, Monografía, n. 1, p. 123-147.

Mora-Á1varez, G., y McDowell, F. W., Miocene volcanism dunng late subduction and early rifting in the Sierra Santa Ursula of westem Sonora, Mexico, in Delgado-Granados, H.; Stock, J. M. Y Aguirre-Oiaz, G., OOs., Cenozoic teetonics and volcanism of western Mexico: Boulder, Colorado, Geological Society of America Special Paper 334, en prensa.

Munguía, L., and Fletcher, M. J., Seismotectonics and distributionof active continental rifting on the southwest margin of the Gulf of Califomia, Mexico: en revisión en Tectonics, junio 1999.

Neuhaus, J. R, Cassidy, M., Krummenacher, D., and Gastil, R. G., 1988, Timing of protogulf extension and transtensional rifting through volcaniclsedimentary stratigraphy of SW Isla Tiburon, Gulf of California, Sonora, Mexico: Geological Society of Amenea Abstract with program, p. 218.

Neuhaus, J., 1989, Volcanic and non-marine stratigraphy of southwest Isla Tiburon, Gulf of Califomia, Mexico: San Diego, Califomia, San Diego State University, Master Thesis, 170 p.

Nourse, A. J.; Anderson, H. T. Y Silver, T. L., 1994, Tertiary metarnorphic core complexes in Sonora, northwestem Mexico: Tectonics, v. 13, p. 1,161-1,182.

Ortega-Gutiérrez, F., Mitre Salazar, L.M., Roldán-Quintana, J., Aranda-Gómez, J., Morán-Zenteno, D., Alaniz-Álvarez, S. A., Nieto-Samaniego, A. F., 1992, Carta Geológica de la República Mexicana escala 1:2,000 000. Universidad Nacional Autónoma de México, Instituto de Geología y Secretaría de Energía, Minas e Industria Paraestatal, Consejo-de Recursos Minerales, 5a. OOición, 1 mapa, 1 folleto, 71 p.

Paz-Moreno, F. A., 1992, Le volcanisme mio-plio-quatemaire de l'etat du Sonora (nord-ouest du Mexique): Evolution Spatiale et Chronologique; implications petrogenetiques: Marseille, France, Universite de Oroit, d'Economie et des Sciences d'AixMarseille, Faculte des Sciences et Techniques de St-Jerome, $211 \mathrm{p}$.
Paz-Moreno, F. A., Y Oemant, A., 1999, The recent Isla San Luis volcanic center-Petrology 01 a rift-relatOO volcanic suite in the northern Gulf of Calilornia, Mexico: Journal 01 Volcanology and Geothermal Research, en prensa.

Parkin, E. L, 1998, Tectonic controls on the Pliocene to Quaternary stratigraphic and structural evolution of Bahía de Guadalupe basin, Baja Calilornia, Mexico: Los Angeles, California, University 01 California Los Angeles, Master Thesis, 122 p.

Rogers, G.; Saunders, A. D.; Terrel, O. J.; Verma, S. P.; y Marriner, J. S., 1985, Geochemestry of Holocene volcanic rocks associatOO with ridge subduction in Baja California, Mexico: Nature, v. 315, p. 389-392.

Saunders, A. D.; Fornari, O. J. y Morrison, M. A., 1982, The composition and emplacement of basaltic magmas produced during the development of continental margin basins-the Gulf of California, Mexico: Journal of the Geological Society of London,v.139,p.335-346.

Saunders, A. D.; Rogers, G.; Marriner, G. F.; Terrell, D. J.; Y Verma, P. S., 1987, Geochemistry of Cenozoic volcanic rocks, Baja California, Mexico: implications for the petrogenesis 01 postsubduction magmas, in Weaver, S. D. and Johnson, R. W., eds., Tectonic Controls on Magma Chemistry: Journal of Volcanology and Geothermal Research, v. 32, n. 1-3, p.223-245.

Sawlan, M. G., 1981, Late Cenozoic volcanism in the Tres Vírgenes area, In: Ortlieb, L., and Roldán-Quintana, J. O0s., Geology of northwestern Mexico and southern Arizona: Field guides and papers, Hermosillo, Sonora, Geological Society of America Cordilleran Section, Annual Meeting, p. 309-319.

Sawlan, M. G., and Smith, J. G., 1984, Petrologic characteristics, age and teetonic setting of Neogene volcanic rocks in northern Baja California Sur, Mexico, in Frizzell, A. V., ed., Geology of the Baja California Peninsula: Tulsa, Oklahoma, Society 01 Economic Paleontologists and Mineralogists, Pacific Section, v. 39, p. $237-251$

Sawlan, M. G., 1991, Magmatic evolution of the Gull 01 Calilornia rift, in J. P. Dauphin, and Simoneit, B. R., eds., The Gulf and Peninsular Province of the Calilornias, American Association of Petroleum Geologists, Memoir, n. 47, p. 301.369.

Severinghaus, J., and Atwater, T., 1990, Cenozoic geometry and thermal state of the subducting slabs beneath North America, in Wernicke, B. P. ed., Basin and Range extensional tectonics near the latitude of Las Vegas, Nevada: Boulder, CO, Geological Society 01 America, Memoir, n. 176, p. 1-22.

Siem, M. E., and Gastil, R. G., 1994, Mid-tertiary to Holocene extension associated with the development 01 the Sierra El Mayor metamorphic core complex, northeastern Baja California, Mexico, in McGill, S. F., and Ross, T. M., eds., Geological investigations of an active margin, Guidebook: ROOlands, California, Geological Society 01 America Cordilleran Section, San Bernardino County Museum Association, p. 107-119.

Spencer, J. E., and Normark, W.R., 1989, Neogene plate tectonics of Baja California Sur continental margin and the southern Gulf of Califomia, Mexico, in Winterer, D., Hussong, M y Decker, R.W., eds, The eastern Pacificand Hawaii: Boulder, Colorado, Geological Society of America, The Geology of North America, v. N, p. 489-498.

Spencer E.J.; Richard, S.M.; Reynolds, S.J.; Miller, R.J.; Shafiqullah, M.; Gilbert, W.G. y Grubensky, M.J., 1995, Spatial and temporal relationship between mid-Tertiary magmatism and extension in southweslern Arizona: Journal of Geophysical Research,v. 100, p. 10,321-10,351.

Stock, J. M., 1989, Sequence and geochronology of miocene rocks adjacent to the main gulf escarpment: southern Valle Chico, Baja Calilornia Norte, Mexico: Geofísica Internacional, v. 28, n. 5, p. 851-896. 
Stock, J. M., Y Hodges, K. V., 1989, Pre-Pliocene extension around tlle Gulf of California and the transfer of Baja California to the Pacific plate: Tectonics, v. 8, p. 99-115.

Stock, J. M., Y Hodges, K. V., 1990, Miocene to recent structural development of an extensional accommodation zone, northeastern Baja California, Mexico: Journal of Structural Geology, v. 12, p. 315-328.

Stock, J. M., Relation of the Puertecitos Volcanic Province, Baja California, Mexico, to the development of tlle plate boundary in the Gulf of California, in Delgado-Granados, H.; Aguirre-Diaz, G., and Stock, J. M., oos., Cenozoic tectonics and volcanism of Mexico, Boulder, $c a$, Geological Society of America Special Paper 334, en prensa.

Stock, J. M., and J. Lee, 1994, Do microplates in subduction zones leave a geological record?: Tectonics, v. 13, p. 1472-1487.

Stock, J. M.; Martín Barajas A., and Téllez-Duarte, M., 1996, Earty rift sedimentation and structure along the N E margin of Baja California, in: Abbott, P, and Cooper, J., eds., American Association of Petroleum Geologists, Field Conference Guide: Pacific Section SEPM, v. 80, p. 337-372.
Suárez-Vidal, F.; Armijo, R.; Morgan, G. Bodin, P., and Gastil, R G., 1991, Framework of recent and active faulting in northern Baja California, in: J. P. Dauphin, and B. R Simoneit, oos., The Gulf and Peninsular Province of the Californias, American Association of Petroleum Geologists, p. 285-300.

Suter, M., 1991, State of stress and active deformation in Mexico and western Central America, in Slemmons, D.B. et al., eds., Neotectonics of North America: Bulder, Colorado, Geological Society of America, Decade of North American Geology, Dseade Map Volume, p. 401-421.

Umhoefer, P.J.; Dorsey, J.R y Renne, P.R., 1994, Tectonics of tlle Pliocene Loreto basin, Baja California Sur, Mexico, and evolution of the Gulf 01 California: Geology, v. 22, p. 646-652.

Umhoeler, P. J., and Dorsey, J. R, 1997, Translation of terranesLessons from central Baja California, Mexico: Geology, v. 25, p. 1,007-1,010.

Zanchi, A., 1994, The opening of tlle Gulf of California near Loreto, Baja California. Mexico-from basin and range extension to transtensional tectonics: Joumal of Structural Geology, v. 16, p. 1,619-1,639. 